\title{
THREE THEOREMS ON CLOSURE OF BIORTHOGONAL SYSTEMS OF FUNCTIONS*
}

\author{
BY R. E. LANGER
}

1. Introduction. Professor Birkhoff has given a theorem $\dagger$ which asserts the closure of a given normal orthogonal set of functions provided it is sufficiently near to another such set which is closed. In its applications this theorem is frequently used in conjunction with asymptotic forms for the functions involved, and the information at hand applies only to a portion of the entire set. For this reason an extension of the theorem to sets which involve a certain lack of closure is useful. Such an extension together with a generalization of the theorem to biorthogonal systems of functions is given below in Theorem I.

In Theorems II and III the discussion is restricted to the closure of such biorthogonal systems as are composed of the characteristic functions of an integral equation.

A set of continuous functions $\left\{u_{n}(x)\right\}$ will be said to involve a $k$-fold lack of closure on a given interval if there exist precisely $k$ continuous functions not identically zero which are linearly independent and are orthogonal on the interval to every function of the set.

Theorem I. Let $\left\{u_{n}(x), v_{n}(x)\right\}$, that is,

$$
\left\{\begin{array}{l}
u_{0}(x), u_{1}(x), u_{2}(x), \cdots, \\
v_{0}(x), v_{1}(x), v_{2}(x), \cdots,
\end{array}\right.
$$

be a normalized biorthogonal system of continuous functio on the interval $a \leqq x \leqq b$, and let $\left\{\bar{u}_{n}(x), \bar{v}_{n}(x)\right\}$, i. e.,

$$
\left\{\begin{array}{c}
\bar{u}_{0}(x), \bar{u}_{1}(x), \bar{u}_{2}(x), \cdots, \\
\bar{v}_{0}(x), \bar{v}_{1}(x), \bar{v}_{2}(x), \cdots,
\end{array}\right.
$$

* Presented to the Society, December 28, 1926.

$\dagger$ Proceedings of the National Academy, vol. 3 (1917), pp. 656-659. 
be a second normalized biorthogonal system which is such that (a) the series

$$
\sum_{n=0}^{\infty}\left[u_{n}(x)-\bar{u}_{n}(x)\right] v_{n}(y), \quad a \leqq x, y \leqq b,
$$

converges to a function $H(x, y)$ less than $1 /(b-a)$ in absolute value; and

(b) the convergence is such that the series upon being multiplied by any continuous function may be integrated term by term as to $x$ to yield a uniformly convergent series.

Then if the set $\left\{u_{n}(x)\right\}$ involves a k-fold lack of closure on the interval $(a, b)$ the lack of closure of the set $\left\{\bar{u}_{n}(x)\right\}$ is at most $k$-fold.*

By hypothesis there exist $k$ linearly independent continuous functions $\phi_{i}(x), i=1,2, \cdots, k$, which are orthogonal to all functions of the set $\left\{u_{n}(x)\right\}$, and every continuous function $\Phi(x)$, which is orthogonal to all functions $u_{n}(x)$, is expressible in the form

$$
\Phi(x)=\sum_{i=1}^{k} c_{i} \phi_{i}(x)
$$

It is clear that the entire set

$$
\phi_{1}(x), \phi_{2}(x), \cdots, \phi_{k}(x), u_{0}(x), u_{1}(x), \cdots
$$

is closed.

Suppose now that there exist $(k+1)$ continuous functions $f_{i}(x), i=1,2, \cdots, k+1$, which are orthogonal to all the functions $\bar{u}_{n}(x)$. Then set

$$
F(x)=\sum_{i=1}^{k+1} \alpha_{i} f_{i}(x)
$$

the $(k+1)$ constants $\alpha_{i}$ being chosen not all zero and so as to satisfy the $k$ linear relations

* A slightly different generalization of Birkhoff's theorem to biorthogonal systems in the case $k=0$ (closure) is given by the author, Transactions of this Society, vol. 28, p. 585 . 


$$
\int_{a}^{b} \Psi(y) \phi_{i}(y) d y=0,(i=1,2, \cdots, k),
$$

where

$$
\Psi(y)=F(y)-\int_{a}^{b} F(x) H(x, y) d x .
$$

Because of hypothesis (b), the function $\Psi(y)$ is continuous. Substituting for $H(x, y)$ the series (3), and observing from (5) that $F(x)$ is orthogonal to all functions $\bar{u}_{n}(x)$, we obtain from (7) the relation

$$
\begin{aligned}
\int_{a}^{b} \Psi(y) & u_{n}(y) d y=\int_{a}^{b} F(y) u_{n}(y) d y \\
& -\sum_{j=0}^{\infty} \int_{a}^{b} F(x) u_{j}(x) d x \int_{a}^{b} v_{j}(y) u_{n}(y) d y .
\end{aligned}
$$

Since the system (1) is normalized biorthogonal, however, the right member of this equation vanishes for all $n$. From this fact and relation (6), it is seen that $\Psi(x)$ is orthogonal to every function of the closed set (4). It follows that $\Psi(y) \equiv 0$, that is,

$$
F(y)=\int_{a}^{b} F(x) H(x, y) d x .
$$

If, now, the maximum numerical value of $F(y)$ on $(a, b)$ is $F$, and if this value is taken on for $y=y_{0}$, we have

$$
\left|\int_{a}^{b} F(x) H\left(x, y_{0}\right) d x\right|=F .
$$

But since $|H(x, y)|<1 /(b-a)$, this is impossible unless $F=0$, and therefore $F(x) \equiv 0$. Hence the $(k+1)$ functions $f_{i}(x)$ must be linearly dependent and the set $\left\{\bar{u}_{n}(x)\right\}$ involves at most a $k$-fold lack of closure.

2. A Theorem in Integral Equations. The theory of the integral equation with a symmetric kernel contains a theorem to the effect that if the system of characteristic functions 
$\left\{u_{n}(x)\right\}$ is not closed then every function which is orthogonal to $u_{n}(x)$ for all $n$ must be also orthogonal to the kernel.* The following theorem is in a sense analogous for the theory of the integral equation with a general kernel. We consider the integral equation

$$
u(x)=\lambda \int_{a}^{b} K(x, \xi) u(\xi) d \xi
$$

in which the kernel is bounded and has its discontinuities regularly distributed. $\dagger$

THEOREM II. If the set of characteristic functions $\left\{u_{n}(x)\right\}$ of equation (8) is not closed but involves only a finite lack of closure, then there always exists at least one continuous function $F(x) \not \equiv 0$ which is orthogonal to the kernel, that is,

$$
\int_{a}^{b} K(x, \xi) F(x) d x=0 .
$$

By hypothesis there exist $k$ linearly independent continuous functions $f_{i}(x), i=1,2, \cdots, k$, such that

$$
\int_{a}^{b} f_{i}(\xi) u_{n}(\xi) d \xi=0
$$

for all values of $n$; and every function orthogonal to all functions $u_{n}(x)$ is expressible linearly in terms of the functions $f_{i}(x)$. Consider the function

$$
\theta_{i}(x)=\int_{a}^{b} K(\xi, x) f_{i}(\xi) d \xi .
$$

Multiplying it by $u_{n}(x)$ and integrating, we find because of (8) and (9) that $\theta_{i}(x)$ is orthogonal to $u_{n}(x)$ for all values of $n$. Hence

$$
\int_{a}^{b} K(\xi, x) f_{i}(\xi) d \xi=\sum_{j=1}^{k} c_{i j} f_{j}(x) .
$$

* T. Lalesco, Théorie des Équations Intégrales, Paris, 1912, p. 69.

$\dagger$ The reader will have no difficulty in observing where restrictions of this kind can be lightened if desired. 
Let the function $F(x)$ be defined now by the relation

$$
F(x)=\sum_{i=1}^{k} \alpha_{i} f_{i}(x) .
$$

Then, from (11), we have

$$
\int_{a}^{b} K(\xi, x) F(\xi) d \xi=\sum_{j=1}^{k} f_{j}(x) \sum_{i=1}^{k} c_{i j} \alpha_{i} .
$$

Now the equation

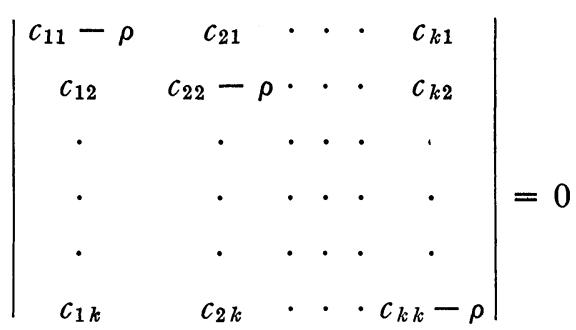

admits of at least one root $\rho=\rho_{1}$; hence we may choose the constants $\alpha_{i}$ in (12) not all zero, and so that

$$
\sum_{i=1}^{k} c_{i j} \alpha_{i}=\rho_{1} \alpha_{j}
$$

Substituting this in (13), we find that

$$
\int_{a}^{b} K(\xi, x) F(\xi) d \xi=\rho_{1} F(x)
$$

Suppose now that $\rho_{1} \neq 0$. Then $F(x)$ is a characteristic function of the associated equation for $\lambda=1 / \rho_{1}$, and it follows that

$$
F(x)=\sum \beta_{l} v_{l}(x)
$$

where the coefficients on the right are constants and the sum includes all the characteristic functions $v_{l}(x)$ of the associated equation which occur in the biorthogonal normal system $\left\{u_{n}(x), v_{n}(x)\right\}$; and which correspond to the value $\lambda=1 / \rho_{1}$. From (12), however, $F(x)$ is orthogonal to all 
functions of the set $\left\{u_{n}(x)\right\}$, and $F(x) \not \equiv 0$ since the functions $f_{i}(x)$ are linearly independent. This contradicts (15), since the system $\left\{u_{n}(x), v_{n}(x)\right\}$ is normalized biorthogonal. It follows that $\rho_{1}=0$, which proves the theorem.

3. A more General Form of Theorem II. For simplicity in the formulation of Theorem III we shall call a function $u(x)$ which satisfies the relation

$$
\int_{a}^{b} K(x, \xi) u(\xi) d \xi=0
$$

a characteristic function of equation (8) for the value $\lambda=\infty$. If the equation (8) admits of $m$ linearly independent characteristic functions $u_{-i}(x), i=1,2, \cdots, m$, for $\lambda=\infty$, and the associated equation also admits of $m$ characteristic functions $v_{-i}(x), i=1,2, \cdots, m$, for $\lambda=\infty$, the system $\left\{u_{-i}(x), v_{-i}(x)\right\}$ may be biorthogonalized and normalized provided no linear combination of the functions of either set $u_{-i}(x)$ or $v_{-i}(x)$, is orthogonal to all the functions of the other set*. If the system $\left\{u_{i}(x), v_{i}(x)\right\}$ is biorthogonal and normal, it follows since

$$
\int_{a}^{b} u_{-i}(x) v_{j}(x) d x=\lambda_{j} \int_{a}^{b} v_{j}(\xi) \int_{a}^{b} K(\xi, x) u_{-i}(x) d x d \xi=0,
$$

that the entire system

$$
\left\{\begin{array}{l}
u_{-m}(x), \cdots, u_{-1}(x), u_{0}(x), u_{1}(x), \cdots \\
v_{-m}(x), \cdots, v_{-1}(x), v_{0}(x), v_{1}(x), \cdots
\end{array}\right.
$$

is biorthogonal and normal. We shall call this the extended system $\left\{u_{n}(x), v_{n}(x)\right\}$, and shall call the entire set of functions $u_{n}(x), n=-m,(-m+1), \cdots$ the extended set $\left\{u_{n}(x)\right\}$.

Theorem III. If the integral equation (8) and its associated equation admit as solutions for $\lambda=\infty$ the functions of a biorthogonal normal system $\left\{u_{-i}(x), v_{-i}(x)\right\}$, and if the

* Goursat, Cours d'A nalyse Mathêmatique, 3d ed. (1923), vol. 3, p. 393. 
extended set $\left\{u_{n}(x)\right\}$ is not closed but involves only a finite lack of closure, then there always exists at least one continuous function $F(x) \not \equiv 0$ which is linearly independent of the functions $v_{-i}(x)$, and which is orthogonal to the kernel, that is,

$$
\int_{a}^{b} K(x, \xi) F(x) d x=0 .
$$

By hypothesis there exist $k$ functions $f_{i}(x), i=1,2, \cdots, k$, which satisfy (9), where $n$ runs over all values in the extended set $\left\{u_{n}(x)\right\}$. Precisely as in the preceding proof it follows that $\theta_{i}(x)$ in $(10)$ is orthogonal to the functions $u_{n}(x)$ for $n=0,1,2, \cdots$ But further

$$
\int_{a}^{b} \theta_{i}(x) u_{-j}(x) d x=\int_{a}^{b} f_{i}(\xi) \int_{a}^{b} K(\xi, x) u_{-j}(x) d x d \xi=0
$$

since $u_{-j}(x)$ is a characteristic function for $\lambda=\infty$. Hence again $\theta_{i}(x)$ is orthogonal to $u_{n}(x)$ for all $n$. As in the preceding proof we may construct now a function $F(x)$ by formula (12) which satisfies equation (14). Moreover the previous reasoning shows that $\rho_{1}=0$, and hence that $F(x)$ is orthogonal to $K(x, \xi)$. To show that $F(x)$ is linearly independent of the functions $v_{-i}(x)$, we assume the contrary, namely that

$$
\gamma_{0} F(x)+\sum_{i=1}^{m} \gamma_{i} v_{-1}(x) \equiv 0
$$

Since the extended set $\left\{u_{n}(x), v_{n}(x)\right\}$ is biorthogonal and normal, while $F(x)$ is orthogonal to every function $u_{n}(x)$, it follows, upon multiplying the relation by $u_{-j}(x)$ and integrating, that $\gamma_{j}=0, j=1,2, \cdots, m$. This implies that $F(x) \equiv 0$ which involves a contradiction.

Corollary. If the system of all linearly independent solutions of equation (8) and its associated equation for $\lambda=\infty$ may be made biorthogonal and normal, and if any subset of the extended set $\left\{u_{n}(x)\right\}$ involves only a finite lack of closure, then the extended set $\left\{u_{n}(x)\right\}$ is closed. 
If a subset involves only a finite lack of closure, the same must be true of the entire set.

4. Examples. The following examples are given to illustrate the necessity of certain parts of the hypotheses which have been made. Thus in Theorem II the case of an infinite lack of closure is excluded. Such a case is represented by the Volterra equation in which $a=0, b=1$, and

$$
K(x, \xi)=\left\{\begin{array}{l}
0 \text { for } \xi>x, \\
1 \text { for } \xi \leqq x .
\end{array}\right.
$$

The equation admits of no characteristic values other than 0 , so the lack of closure is infinite. Since

$$
\int_{0}^{1} K(x, \xi) f(x) d x=\int_{0}^{x} f(x) d x=0
$$

implies $f(x) \equiv 0$, the assertion of the theorem clearly does not hold.

In the Corollary to Theorem III, the case in which the totality of solutions for $\lambda=\infty$ cannot be made biorthogonal is excluded. This case is illustrated by the example*

where

$$
K(x, \xi)=K_{1}(x, \xi)+K_{2}(x, \xi),
$$

$$
\begin{aligned}
& K_{1}(x, \xi)=\phi_{1}(x) \phi_{2}(\xi), \\
& K_{2}(x, \xi)=\sum_{n=3}^{\infty} \frac{\phi_{n}(x) \phi_{n}(\xi)}{\lambda_{n}},
\end{aligned}
$$

the set $\left\{\phi_{n}(x)\right\}$ being any closed normalized orthogonal set and the series for $K_{2}(x, \xi)$ converging uniformly. The functions $K_{1}(x, \xi)$ and $K_{2}(x, \xi)$ are orthogonal, and hence the set of characteristic functions for $K(x, \xi)$ is composed of those for $K_{1}(x, \xi)$ and $K_{2}(x, \xi) . \dagger$ The characteristic functions for $K_{2}(x, \xi)$ are obviously $\phi_{n}(x), n=3,4, \cdots$. On the other hand equation

* This example was given to me by Professor J. Tamarkin of Dartmouth College.

† Goursat, loc. cit., p. 402. 
is of the form

$$
u(x)=\lambda \int_{a}^{b} K_{1}(x, \xi) u(\xi) d \xi
$$

$$
u(x)=\lambda \int_{a}^{b} \phi_{2}(\xi) u(\xi) d \xi \phi_{1}(x)=c \phi_{1}(x),
$$

whereas the substitution of this value in the equation yields

$$
c \phi_{1}(x)=\lambda c \phi_{1}(x) \int_{a}^{b} \phi_{2}(\xi) \phi_{1}(\xi) d \xi=0 .
$$

Hence $k_{1}(x, \xi)$ admits of no characteristic functions. Further if $f(x)$ is a characteristic function for $\lambda=\infty$ we have upon setting

the relation

$$
\int_{a}^{b} f(\xi) \phi_{1}(\xi) d \xi=f_{i}
$$

$$
\int_{a}^{b} K(x, \xi) f(\xi) d \xi=f_{2} \phi_{1}(x)+\sum_{n=3}^{\infty} \frac{f_{n} \phi_{n}(x)}{\lambda_{n}}=0 .
$$

Multiplying this by $\phi_{j}(x)$ and integrating we find that $f_{j}=0$ for $j=2,3, \cdots$, whence $f(x)$ must be of the form $f(x)=$ $c \phi_{1}(x)$ and $\phi_{1}(x)$ is the only characteristic function for $\lambda=\infty$. In similar fashion it is seen that $\phi_{2}(x)$ is the only characteristic function of the associated equation for $\lambda=\infty$. Since on the one hand $\phi_{1}(x)$ and $\phi_{2}(x)$ are orthogonal the hypothesis of the corollary cannot be satisfied. On the other hand since the entire set of characteristic functions

$$
\phi_{1}(x), \phi_{3}(x), \phi_{4}(x), \cdots
$$

is not closed while the subset $\phi_{i}(x), i=3,4, \cdots$, involves only a two-fold lack of closure the assertion of the theorem clearly fails.

\section{BROWN UNIVERSITY}

